\title{
Competition between Plasmid-Bearing and Plasmid-Free Organisms in the Host: Population Dynamics and Antibiotic Resistance
}

\author{
Hui-Xing Songa, b Yuan-Ying Peng a Zhi-Fu Zhu ${ }^{\mathrm{c}}$ \\ a School of Life Science, Qufu Normal University, Qufu, Shandong, ${ }^{b}$ School of Life Science, Southwest University, \\ Key Laboratory of Eco-Environments in Three Gorges Reservoir Region (Ministry of Education), Chongqing, and \\ 'Department of Mathematics, Chongqing Communication Institute, Chongqing, PR China
}

\section{Key Words}

Plasmid-bearing organisms • Plasmid-free organisms •

Resistance to antibiotics

\begin{abstract}
Objective: To clarify the effects of antibiotic exposure on bacterial antibiotic resistance. Materials and Methods: A competitive population dynamics model for plasmid-bearing organisms and plasmid-free organisms in the host was established. The Poincaré-Bendixson theorem was used for analysis. Results: All possible results of the competition dependent on biologically meaningful parameters were obtained, which can be classified into eight categories. With the outcomes of the plasmid model, we discussed the sterilizing effect if an antibiotic was added to the host. Conclusion: When antibiotic-resistant bacteria existed in a host, a higher dosage of antibiotics was needed with an increased risk of survival of plasmid-bearing organisms and greater danger of resistance to antibiotics.
\end{abstract}

Copyright $\odot 2006$ S. Karger AG, Basel
(C) 2006 S. Karger AG, Basel

1011-7571/06/0156-0436\$23.50/0

Fax +41 613061234

E-Mail karger@karger.ch

www.karger.com
Accessible online at: www.karger.com/mpp

\section{Introduction}

With the development and widespread use of antibiotics, dependence has increased tremendously, especially since many clinicians tend to prescribe antibiotics for inflammatory reactions. Antibiotic use is often blamed for an increase in the prevalence of infections due to antibiotic-resistant bacteria and for shortening the life of natural antibiotics, leading to an apparently inexorable advent of a postantibiotic era [1-3]. Theoretical discussions in the literature have examined antibiotic therapy and resistance in order to clarify the effects of antibiotic exposure on bacterial antibiotic resistance and postpone the advent of a postantibiotic era [3-8].

Genetically altered organisms frequently occur in a host, for example, the human body. This alteration is accomplished by the insertion into the cell of a plasmid, a piece of DNA that replicates within the cell independently of chromosomal DNA. This genetic material is reproduced when the cell divides. Similarly, the plasmid can be lost in the reproductive process. The plasmid-bearing organism is likely to be a lesser competitor than the plasmid-free organism because of the added load on its metabolic machinery. To compensate, an additional piece of 
genetic material is added to the plasmid, one that codes for resistance to an inhibitor (an antibiotic) and the inhibitor is added to the host by injection, orally or by transfusion.

Many papers discuss plasmid models in chemostat, for instance based on the mass balances of the organisms, i.e. the competitive model between the plasmid-bearing and the plasmid-free organisms without an inhibitor $[9,10]$, and the competitive model between the plasmid-bearing and the plasmid-free organisms with an inhibitor $[11,12]$. In this paper, based on the above-mentioned mechanism of bacterial antibiotic resistance within the host and the competition in terms of the metabolic cost of carrying the plasmid, we establish a competitive population dynamics model to explore the competitive interactions between two populations of bacteria: one with a plasmid that confers resistance to antibiotics and another without the plasmid. With the results of the plasmid model, we analyze the sterilizing effect when an antibiotic is added to the host by injection, orally or by transfusion. Those analyses effectively explain the relationship between antibiotic resistance and the dosage of antibiotic exposure. Plasmid models have been cited by a significant number of researchers [13-18].

\section{Description of the Model}

The competitive population dynamics model between the plasmid-bearing and the plasmid-free organisms without an inhibitor, modified from the model of Wang et al. [8], Hsu et al. [10] and Zhiqi and Hadeler [11], is described as

$$
\begin{aligned}
& \dot{x}_{1}=x_{1}\left[r_{1}(1-q)-\delta_{11} x_{1}-\delta_{12} x_{2}\right], \\
& \dot{x}_{2}=x_{2}\left[r_{2}-\delta_{21} x_{1}-\delta_{22} x_{2}\right]+q r_{1} x_{1},
\end{aligned}
$$

where $x_{1}(t)$ denotes the concentration of plasmid-bearing organisms at time $t$, and $x_{2}(t)$ is the concentration of plasmid-free organisms. The specific growth rates and death rates of plasmid-bearing and plasmid-free organisms are $r_{1}, r_{21}, \delta_{11} x_{1}$ and $\delta_{22} x_{2}$, respectively. Here, $q$ is the probability that a plasmid is lost in reproduction, and hence $0<q<1 . \delta_{i j}$ is the competition coefficient of the $i$-th organism for the $j$-th organism. For biological consistency, all parameters are positive constants, and the initial value of system 1 is $x_{i}(0)>0, i=1,2$.

System 1 shows that each species in isolation would exhibit logistic growth. That is, when system 1 is considered restricted to the $i$-th coordinate axis, we have $\dot{x}_{i}=$ $x_{i}\left[r_{i}-\delta_{i j} x_{i}\right], a_{i}, \delta_{i i}>0$, in which the repulsion at 0 (growth of small populations) and the repulsion at $\bullet$ (competition within large populations) balance at an attracting fixed point, $R_{i}$, at the carrying capacity $r_{i} / \delta_{i i}$. However, from the following description, it is found that there are more complicated phenomena when the species are not isolated.

\section{Dynamics of the Plasmid Model}

First, we will show that system 1 is reasonable. That is, we will prove that every solution of system 1 is always positive and ultimately bounded for any initial value.

Because $x_{1}=0$ is the constant solution of system 1 , by the uniqueness of initial value problems and continuous dependence on the initial value, we get $x_{1}(t)>0$ for any $t \geq 0$, i.e. $x_{1}(t)$ is always positive. If the first time $x_{2}(t)=$ 0 and $t_{1}>0$, then $\dot{x}_{2}\left(t_{1}\right)=q r_{1} x_{1}\left(t_{1}\right)>0$, meaning $x_{2}(t)<$ 0 for $t \in\left(t_{1}-\varepsilon, t_{1}\right), \varepsilon$ being an arbitrarily small positive constant, a contradiction. Thus, $x_{2}(t)$ is always positive. Now, by the first equation of system 1 , we have

$$
\dot{x}_{1}=x_{1}\left[r_{1}(1-q)-\delta_{11} x_{1}-\delta_{12} x_{2}\right]<x_{1}\left[r_{1}(1-q)-\delta_{11} x_{1}\right],
$$

so by the comparison principle,

$$
\lim _{t \rightarrow+\infty} x_{1}(t) \leq \frac{r_{1}(1-q)}{\delta_{11}}=x_{1}^{\infty} ;
$$

by the second equation of system 1 , we have

$$
\dot{x}_{2}=x_{2}\left[r_{2}-\delta_{21} x_{1}-\delta_{22} x_{2}\right]+q r_{1} x_{1}<x_{2}\left[r_{2}-\delta_{22} x_{2}\right]+\frac{q r_{1}^{2}(1-q)}{\delta_{11}},
$$

so

$$
\lim _{t \rightarrow+\infty} x_{2}(t) \leq \frac{r_{2}+\sqrt{r_{2}^{2}+\frac{4 \delta_{22} q r_{1}^{2}(1-q)}{\delta_{11}}}}{2 \delta_{22}}=x_{2}^{\infty} .
$$

Therefore, the above conclusion is correct and the region $\Omega=\left\{\left(x_{1}, x_{2}\right) \mid 0<x_{1} \leq x_{1}^{\bullet \bullet}, 0<x_{2} \leq x_{2}^{\bullet}\right\}$ is a positively invariant region of system 1 . For the convenience of the description, we will restrict our analysis to region $\Omega$ for system 1 .

We use the following notation for the relevant rest points of system $1, E=\left(x_{1}^{*}, x_{2}^{*}\right)$. A rest point of system 1 does not exist if any one of its components is negative, that is, a rest point is the nonnegative solution of the algebraic equations as follows

$$
\begin{aligned}
& x_{1}\left[r_{1}(1-q)-\delta_{11} x_{1}-\delta_{12} x_{2}\right]=0, \\
& x_{2}\left[r_{2}-\delta_{21} x_{1}-\delta_{22} x_{2}\right]+q r_{1} x_{1}=0 .
\end{aligned}
$$



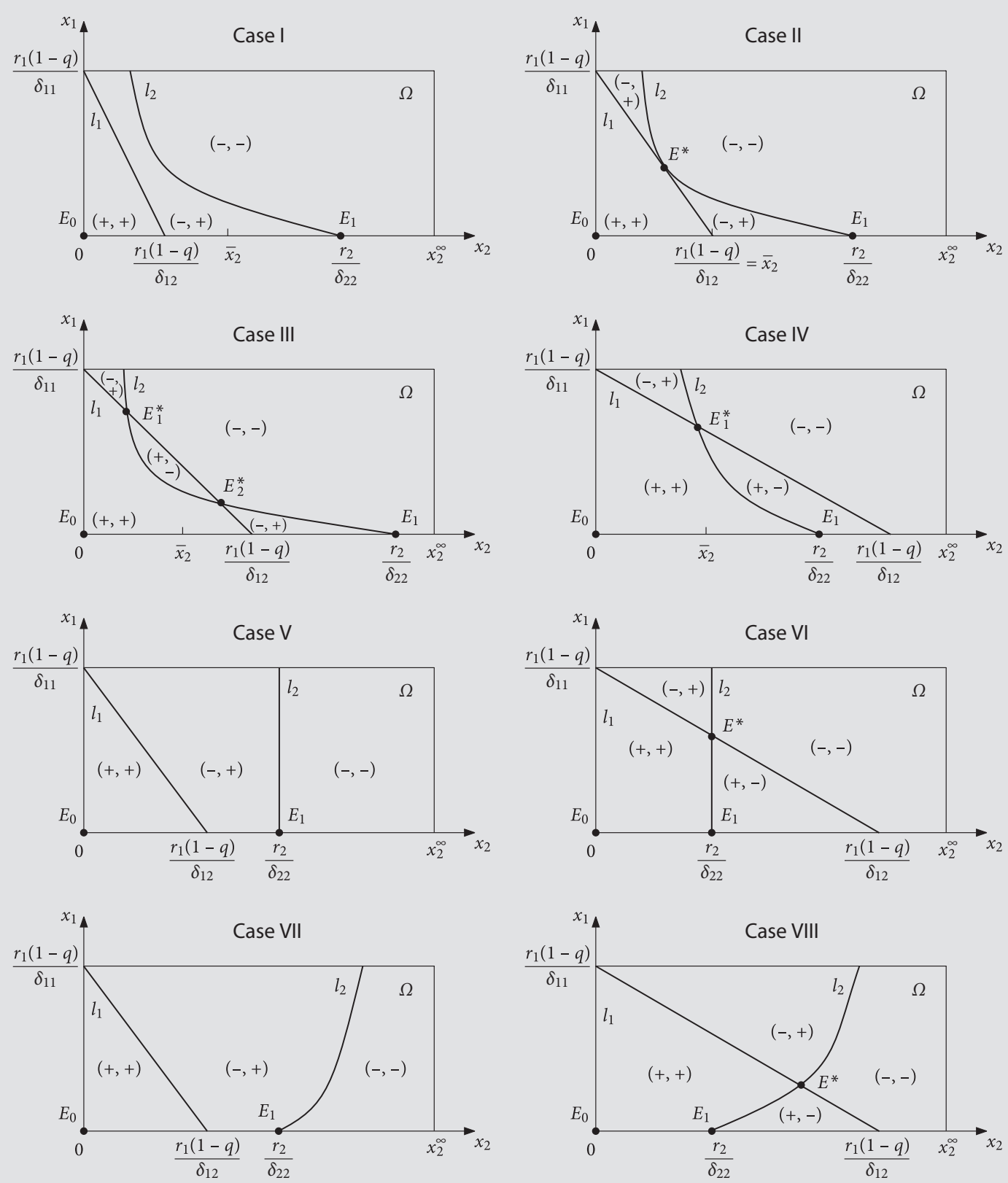

Fig. 1. Illustration of the rest points and the vector field of system 1 . Each rest point is represented by a closed $\operatorname{dot}(\bullet)$, and the sign of $\dot{x}_{1}, \dot{x}_{2}$ in each subregion is denoted by $(a, b)$, where $a$ is the sign of $\dot{x}_{1}$, and $b$ is the sign of $\dot{x}_{2}$. Cases I-IV: $\frac{q r_{1}}{\delta_{21}}<\frac{r_{2}}{\delta_{22}}$; cases V and VI: $\frac{q r_{1}}{\delta_{21}}=\frac{r_{2}}{\delta_{22}}$; cases VII and VIII: $\frac{q r_{1}}{\delta_{21}}>\frac{r_{2}}{\delta_{22}}$; where $\bar{x}_{2}$ is the largest root of the equation $\left[\frac{r_{1}\left(1-q\left(q r_{1}-\delta_{21} \bar{x}_{2}\right)\right)}{\delta_{11} \bar{x}_{2}}-r_{2}\right]^{2}-\frac{4 \bar{x}_{2} r_{1}(1-q)\left(r_{2} \delta_{21}-q r_{1} \delta_{11}\right)}{\delta_{11} \bar{x}_{2}}=0$. 
Table 1. The asymptotical stabilizability of the rest points under different criteria

\begin{tabular}{|c|c|c|c|c|}
\hline Case & \multicolumn{2}{|c|}{$\begin{array}{l}\text { Criteria for existence of the rest } \\
\text { points }\end{array}$} & Rest points & Stabilizability \\
\hline I & \multirow{4}{*}{$\frac{q r_{1}}{\delta_{21}}<\frac{r_{2}}{\delta_{22}}$} & $\frac{r_{1}(1-q)}{\delta_{12}}<\bar{x}_{2}$ & $\left\{E_{0}, E_{1}\right\}$ & $E_{1}$, global stable \\
\hline II & & $\frac{r_{1}(1-q)}{\delta_{12}}<\bar{x}_{2}$ & $\left\{E_{0}, E_{1}, E^{*}\right\}$ & $\begin{array}{l}E_{1}, E^{*} \text {, dependent on } \\
\text { the initial value }\end{array}$ \\
\hline III & & $\bar{x}_{2}<\frac{r_{1}(1-q)}{\delta_{12}}<\frac{r_{2}}{\delta_{22}}$ & $\left\{E_{0}, E_{1}, E_{1}^{*}, E_{2}^{*}\right\}$ & $\begin{array}{l}E_{1}, E_{1}^{*} \text {, dependent on } \\
\text { the initial value }\end{array}$ \\
\hline IV & & $\frac{r_{1}(1-q)}{\delta_{12}} \geq \frac{r_{2}}{\delta_{22}}$ & $\left\{E_{0}, E_{1}, E_{1}^{*}\right\}$ & $E_{1}^{*}$, global stable \\
\hline $\mathrm{V}$ & \multirow{2}{*}{$\frac{q r_{1}}{\delta_{21}}=\frac{r_{2}}{\delta_{22}}$} & $\frac{r_{1}(1-q)}{\delta_{12}} \leq \frac{r_{2}}{\delta_{22}}$ & \multirow{2}{*}{$\begin{array}{l}\left\{E_{0}, E_{1}\right\} \\
\left\{E_{0}, E_{1}, E^{*}\right\}\end{array}$} & \multirow{2}{*}{$\begin{array}{l}E_{1}, \text { global stable } \\
E^{*}, \text { global stable }\end{array}$} \\
\hline VI & & $\frac{r_{1}(1-q)}{\delta_{12}}>\frac{r_{2}}{\delta_{22}}$ & & \\
\hline VII & \multirow{2}{*}{$\frac{q r_{1}}{\delta_{21}}>\frac{r_{2}}{\delta_{22}}$} & $\frac{r_{1}(1-q)}{\delta_{12}} \leq \frac{r_{2}}{\delta_{22}}$ & $\left\{E_{0}, E_{1}\right\}$ & $E_{1}$, global stable \\
\hline VIII & & $\frac{r_{1}(1-q)}{\delta_{12}}>\frac{r_{2}}{\delta_{22}}$ & $\left\{E_{0}, E_{1}, E^{*}\right\}$ & $E^{*}$, global stable \\
\hline
\end{tabular}

Obviously, $E_{0}=(0,0)$ is the washout rest point, meaning that both organisms become extinct. There is only one possible rest point involving plasmid-free organisms but no plasmid-bearing organisms, denoted

$$
E_{1}=\left(0, \frac{r_{2}}{\delta_{22}}\right) \text {. }
$$

The mixed culture rest point is denoted $E^{*}=\left(x_{1}^{*}, x_{2}^{*}\right)$, where $E^{*}$ is the point of intersection between curve $l_{1}$ : $r_{1}(1-q)-\delta_{11} x_{1}-\delta_{12} x_{2}=0$ and $l_{2}: x_{2}\left[r_{2}-\delta_{21} x_{1}-\delta_{22} x_{2}\right]+$ $q r_{1} x_{1}=0$. There are eight cases (fig. 1).

The object of the next discussion is to study the asymptotical stabilizability of the rest points. Since $l_{1}$ and $l_{2}$ are the isocline equations of system $1, l_{1}$ and $l_{2}$ divide region $\Omega$ into several subregions, and $\dot{x}_{1}, \dot{x}_{2}$ keep a fixed sign in each subregion as indicated in figure 1 . We apply the Dulac criterion [18] with the auxiliary function

$$
B\left(x_{1}, x_{2}\right)=\frac{1}{x_{1} x_{2}}
$$

to the vector field given by system 1 . An easy computation in region $\Omega$ yields

$$
\frac{\partial B\left(x_{1}, x_{2}\right) \dot{x}_{1}}{\partial x_{1}}+\frac{\partial B\left(x_{1}, x_{2}\right) \dot{x}_{2}}{\partial x_{2}}=-\left(\frac{\delta_{11}}{x_{2}}+\frac{\delta_{22}}{x_{1}}+\frac{q r_{1}}{x_{2}^{2}}\right)<0 .
$$

Hence there are no nontrivial periodic solutions. Then, using the Poincaré-Bendixson theorem, and with the help of the fixed sign in each subregion, as indicated in figure 1, we obtain the asymptotical stabilizability of the rest points as shown in table 1 .

Using Matlab software, we numerically solved system 1 for each case described (fig. 2), and these simulations also validated the asymptotical stabilizability of the rest points.

\section{Discussion}

The results of the model can be considered from three viewpoints: (1) the stability of the population dynamics, (2) the bifurcation of the rest point, and (3) the antibiotic resistance. 


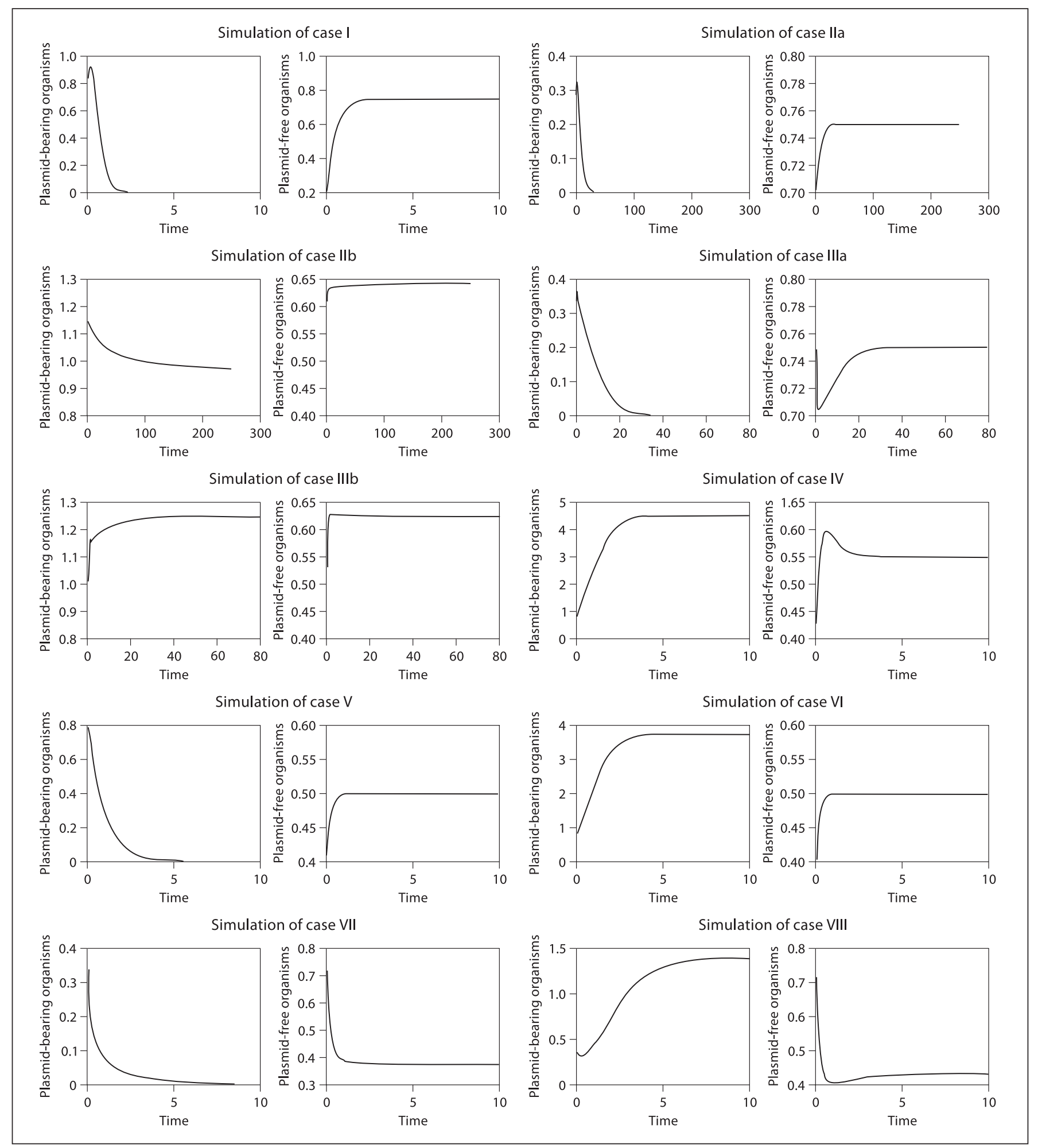

Fig. 2. Illustration of the numerical simulation results of system 1 for each case, with the parameters: $r_{1}=5, r_{2}=3, q=0.2, \delta_{11}=0.4$, $\delta_{21}=2$. Case I: $\delta_{12}=10, \delta_{22}=4$, and initial value $x_{1}(0)=0.8$, $x_{2}(0)=0.4$. Case II: $\delta_{12}=5.62229, \delta_{22}=4$, and initial value $x_{1}(0)=$ $0.4, x_{2}(0)=0.8$ in case IIa and initial value $x_{1}(0)=0.8, x_{2}(0)=$

0.4 in case IIb. Case III: as for case II, except $\delta_{12}=5.6$. Case IV: as for case I, except $\delta_{22}=4$. Case V: as for case I, except $\delta_{22}=6$. Case VI: as for case V, except $\delta_{12}=5$. Case VII: $\delta_{12}=12, d_{22}=8$ and initial value $x_{1}(0)=0.4, x_{2}(0)=0.8$. Case VIII: as for case VII, except $\delta_{12}=8$. 


\section{Population Dynamics}

From table 1, we know that plasmid-bearing organisms will ultimately tend to become extinct and plasmidfree organisms will tend to the carrying capacity

$$
\frac{r_{2}}{\delta_{22}}
$$

for any initial value in cases I, VI and VII. The competition between plasmid-bearing and plasmid-free organisms in the host results in the victory of the plasmid-free organisms. In cases IV, V and VIII, the plasmid-bearing organisms and the plasmid-free organisms will ultimately tend to a positive rest point, $E^{*}$, for any initial value. They will stabilize survival in the host. These are common conclusions that most people can easily think of, and they are also confirmed by the plasmid models in chemostat. Since we have stated that there cannot exist nontrivial periodic solutions, what special phenomena will be found in our model? There are, for example, cases II and III, in which plasmid-bearing organisms tend to become extinct or survive, depending on the initial value of the population: if the initial value of plasmid-bearing organisms is sufficiently small, they will tend to become extinct; otherwise, they will survive in the host. The reason that this special phenomenon occurs is the bifurcation of the rest point, when

$$
\frac{q r_{1}}{\delta_{21}}<\frac{r_{2}}{\delta_{22}}
$$

which is described below.

$$
\begin{aligned}
& \text { Bifurcation of the Rest Point } \\
& \text { In figure 1, if } \\
& \frac{q r_{1}}{\delta_{21}}<\frac{r_{2}}{\delta_{22}}
\end{aligned}
$$

when $r_{1}$ is sufficiently small so that

$$
\frac{r_{1}(1-q)}{\delta_{12}}<\bar{x}_{2},
$$

we can easily observe that there are only two rest points, $E_{0}$ and $E_{1}$, and $E_{1}$ is global asymptotical stability (case I). As $r_{1}$ increases so that

$$
\bar{x}_{2}=\frac{r_{1}(1-q)}{\delta_{12}},
$$

a positive rest point $\left(E^{*}\right)$, appears, it is possible for $E_{1}$ and $E^{*}$ to be stable under a different initial value (case II). Let $r_{1}$ continue to increase so that

$$
\bar{x}_{2}<\frac{r_{1}(1-q)}{\delta_{12}}<\frac{r_{2}}{\delta_{22}}
$$

the bifurcation occurs at this point, the positive rest point, $E^{*}$, becomes two positive rest points $E_{1}^{*}$ and $E_{2}^{*}$, and $E_{1}^{*}$ is a stable node, and $E_{2}^{*}$ is a saddle. There is a conjunctive saddle-node curve between $E_{1}^{*}$ and $E_{2}^{*}$, and it is possible for $E_{1}$ and $E_{1}^{*}$ to be stable under a different initial value (case III). When $r_{1}$ becomes large enough such that

$$
\frac{r_{2}}{\delta_{22}} \leq \frac{r_{1}(1-q)}{\delta_{12}},
$$

the positive rest point $E_{2}^{*}$ will be combined with $E_{1}$, so the stability of $E_{1}$ changes from stable to unstable, and the positive rest point $E_{1}^{*}$ approaches global asymptotical stability (case IV). Therefore, because of the bifurcation of the rest point, the plasmid-bearing organisms will change from a tendency toward extinction to possible survival, and then to final survival.

\section{Antibiotic Resistance}

As described in the Introduction, an inhibitor (an antibiotic) is added to the host by injection, orally or by transfusion in clinical pharmacotherapy. The antibiotics only restrain the growth rate of the plasmid-free organisms and have no influence on the plasmid-bearing organisms. Suppose $p$ is the concentration of the antibiotics, hence $p>0$; the function $e^{-\mu p}$ represents the degree of inhibition of $p$ on the growth rate of the plasmid-free organisms, $r_{2}$, where $\mu$ is a positive constant. Thus, in model 1, the growth rate of the plasmid-free organisms will change from $r_{2}$ to $r_{2} e^{-\mu p}$.

Next, we analyze the aftereffect of the antibiotics. Along with the increase of $p$, and the concentration of the antibiotics, the fraction

$$
\frac{r_{2} e^{-\mu p}}{\delta_{22}}
$$

will become smaller; consequently the inequality

$$
\frac{q r_{1}}{\delta_{21}}<\frac{r_{2} e^{-\mu p}}{\delta_{22}}
$$

will become

$$
\frac{q r_{1}}{\delta_{21}} \geq \frac{r_{2} e^{-\mu p}}{\delta_{22}} .
$$

Similar to figure 1 , when

$$
\frac{q r_{1}}{\delta_{21}}<\frac{r_{2} e^{-\mu p}}{\delta_{22}},
$$


if $p$ increases, a change in sequence occurs from case I to II, to III and to IV. Therefore, the plasmid-bearing organisms will change from extinction to possible survival, and to final survival if

$$
\frac{q r_{1}}{\delta_{21}}=\frac{r_{2} e^{-\mu p}}{\delta_{22}}, \frac{q r_{1}}{\delta_{21}}>\frac{r_{2} e^{-\mu p}}{\delta_{22}}
$$

promptly when $p$ is enhanced. Now, let

$$
\frac{q r_{1}}{\delta_{21}}>\frac{r_{2} e^{-\mu p}}{\delta_{22}}
$$

if $p$ is enhanced. There is a change in sequence from case VII to VIII. Hence, the plasmid-bearing organisms will change from extinction to final survival.

In brief, along with the increase of $p$, the concentration of the antibiotics, the resistance factor will ultimately survive. In other words, if antibiotic-resistant bacteria exist in the host, the dosage of antibiotics is larger, thus there is a greater possibility of survival of plasmid-bearing organisms, and there is more danger of resistance to antibiotics. In order to inhibit resistance to antibiotics, the dosage must be strictly controlled when drug therapy is used in clinics, otherwise resistance to antibiotics is likely to occur, with the postantibiotic era swiftly following.

\section{Acknowledgements}

We would like to thank the referees for their suggestions which have helped improve the clarity of the paper. This work was supported by the National Science Foundation of P.R. China (grant No. 10101029).

\section{References}

1 Berkowitz FE: Antibiotic resistance in bacteria. South Med J 1995;88:797-804.

-2 Tenover FC, Hughes JM: The challenges of emerging infectious diseases: development and spread of multiply-resistant bacterial pathogens. JAMA 1996;275:300-304.

3 Garber AM: Antibiotic exposure and resistance in mixed bacterial populations. Theor Popul Biol 1987;32:326-346.

4 Austin DJ, Kakehashi M, Anderson RM: The transmission dynamics of antibiotic-resistant bacteria: the relationship between resistance in commensal organisms and antibiotic consumption. Proc Biol Sci 1997;264: 1629-1638.

5 Payne RJH, Jansen VAA: Understanding bacteriophage therapy as a density-dependent kinetic process. J Theor Biol 2001;208: 37-48.

6 Peck SL: Antibiotic and insecticide resistance modeling - is it time to start talking? Trends Microbiol 2001;9:286-292.
7 Vinks AA: The application of population pharmacokinetic modeling to individualized antibiotic therapy. Int J Antimicrob Agents 2002;19:313-322.

8 Wang Kai-Fa, Fan Ai-Jun, Deng Guo-Hong Analysis of dynamics on plasmid-bearing and plasmid-free bacteria under the influence of antibiotics. Chin J Antibiot 2004;29: 542-544.

-9 Ryder DF, Dibiasio D: An operational strategy for unstable recombinant DNA cultures. Biotechnol Bioeng 1984;26:942-947.

10 Hsu SB, Waltman P, Wolkowicz GSK: Global analysis of a model of plasmid-bearing, plasmid-free competition in a chemostat. J Math Biol 1994;32:731-742.

11 Zhiqi Lu, Hadeler KP: Model of plasmidbearing, plasmid-free competition in the chemostat with nutrient recycling and an inhibitor. Math Biosci 1998;148:147-159.

12 Shangbing AI: Periodic solutions in a model of competition between plasmid-bearing and plasmid-free organisms in a chemostat with an inhibitor. J Math Biol 2001;42:7194.
13 Stephanopoulis G, San KY, Davidson BH: A novel bioreactor-cell precipitator combination for high-cell density, high flow fermentations. Biotechnol Prog 1985;4:250-259.

14 Lenski RE, Hattingh S: Coexistence of two competitors in one resource and one inhibitor: a chemostat model based on bacteria and antibiotics. J Theor Biol 1986;122:83-93.

15 Simonsen L: The existence conditions for bacterial plasmids: theory and reality. Microb Ecol 1991;22:187-205.

16 Macken CA, Levin SA, Walttatter SA: The dynamics of bacteria-plasmid systems. Math Biosci 1994;32:123-145.

17 Hsu SB, Luo TK, Waltman P: Competition between plasmid-bearing organisms in a chemostat with an inhibitor. J Math Biol 1995;34:225-238.

18 Stephanopoulis G, Lapidus G: Chemostat dynamics of plasmid-bearing plasmid-free mixed recombinant cultures. Chem Eng Sci 1988;43:49-57. 